\section{The IOERT IntraLine accelerator - the development, current state, and future plans}

Przemysław Adrich,

Radosław Hanke, Ewa Kulczycka, Konrad Kosiński, Bartosz Meglicki, Agnieszka Misiarz, Eugeniusz Pławski, Marcin Staszczak, Kaja Swat, Agnieszka Syntfeld-Każuch, Marek Wójtowicz, Anna Wysocka-Rabin

\begin{abstract}
Intraoperative electron radiation therapy (IOERT) is a cancer treatment method that combines oncologic surgery with electron beam radiotherapy. This method can reduce the duration of entire tumor treatment and increase its effectiveness. Moreover, shortening the treatment time significantly reduces the cost and accessibility of the therapy. As a result of interdisciplinary research, an innovative accelerator for IOERT, the IntraLine, was developed. In the course of this work, four patent applications were filed. Today, the work is half way through. In the near future, the device will be optimized. New mechanical design solutions will be developed. Mechanical optimization will significantly reduce the weight of the device. Accelerators control system, which today is in the demo phase, will also be significantly upgraded. This paper describes the stages of the IntraLine accelerator development, its current state and plans for the future R\&D work, within the scope of our new Intra-Dose project.
\end{abstract}

Key words: radiotherapy $\bullet$ mobile electron accelerator $\bullet$ intraoperative electron radiation therapy (IOERT)

P. Adrich ${ }^{\varpi}$, R. Hanke, E. Kulczycka, K. Kosiński, B. Meglicki, A. Misiarz, E. Pławski, M. Staszczak, K. Swat, A. Syntfeld-Każuch, M. Wójtowicz, A. Wysocka-Rabin

Nuclear Techniques \& Equipment Department, National Centre for Nuclear Research (NCBJ), 7 Andrzeja Sołtana Str., 05-400 Otwock/Świerk, Poland, Tel.: +48 22273 1412, Fax: +48 22779 3481,

E-mail: Przemyslaw.Adrich@ncbj.gov.pl

Received: 8 September 2014

Accepted: 31 July 2015

\section{Introduction}

Intraoperative electron radiation therapy (IOERT) is a relatively novel oncological treatment technique. It combines cancer surgery with electron beam irradiation of the tumor site performed right after resection of the tumor. The idea of the IOERT is to destroy with a high dose of radiation any cancerous cells that could potentially remain in the tissues directly neighboring the resected tumor. In this technique, the tumor is first resected and the tumor site is irradiated with a high dose (10 Gy or more) of high energy (above $4 \mathrm{MeV}$ ) electron radiation. The irradiation time is very short, about 1 to 2 minutes. The electron beam is collimated with a circular applicator of a diameter corresponding to the size of the tumor site. The applicator is inserted right into the tumor lodge inside the patient body and restricts the radiation to this area only so that the healthy tissues outside the irradiation field are protected. The penetration depth of the electron radiation is controlled by setting the beam energy appropriately. In this respect, the usage of electrons instead of high-energy gamma radiation is superior as it allows sparing the deeper lying tissues. After completion of the irradiation, the surgical operation is finished.

Up to now, this technique is recommended by the European (GEC-ESTRO) and the American (ASTRO) societies for radiation oncology as a standard of care only for a limited range of certain breast cancer cases $[1,2]$. However, there is a growing 
interest in applying the IOERT also for other kinds and locations of tumors and the results of clinical studies made so far are very promising. Apart from good clinical outcomes, the economy of the whole procedure is an important driver behind the development of the IOERT, as this treatment technique allows significantly shortening or eliminating the external beam-fractionated radiotherapy that normally often follows the oncological surgery. This is beneficial both for the patient and for the clinic.

The IOERT irradiation can be best performed with a mobile electron accelerator designed to work under regime of an operating room. This puts a lot of constraints on the construction of such a machine. In particular, an optimal accelerator should offer a wide range of beam energies and also allow delivering the beam to any location of the surgical site from the clinically most convenient direction. Those requirements are difficult to meet simultaneously. So far, there are only three models of commercially available accelerators dedicated for the IOERT. The Italian LIAC and Novac are relatively lightweight and mobile but offer limited energy range. The American Mobetron offers a wide energy range but has a rather restricted mobility.

To overcome the limitations of the current machines and to offer wider choice for potential future users, a research and development programme aimed at constructing a new accelerator for the IOERT has been initiated at the National Centre for Nuclear Research. In the first phase of the programme, realized within the scope of the project "Development of dedicated systems based on accelerators and detectors of ionizing radiation for medical therapy and in detection of hazardous materials and toxic wastes" (in what follows referred to as AiD) [3], we developed a technology demonstrator that is currently being tested. In the second phase, we aim at constructing a precommercial prototype. In this paper, the design process and the current state of the development is discussed as well as an outlook to the future plans is provided.

\section{Development of the robot}

As mentioned above, one of the challenges in constructing an accelerator dedicated for the IOERT is adequate maneuverability of the machine. To tackle this problem, the accelerator for the IOERT has to have a very different construction than linacs for an external beam therapy. In the latter machines, the location of the isocenter is fixed in space and the arm of the accelerator can only revolve around one axis. The patient has to be positioned on a table such that the target volume is in the isocenter. The philosophy of the application of the IOERT irradiation is very different. There is no fixed isocenter; instead, the machine has to approach the patient and the beam has to be delivered from a direction specific to the location of the surgical site and patient anatomy. To accomplish this, the machine has to move around the surgical table and its radiative head has to have many degrees of freedom.
Perhaps the simplest solution could be attaching a linac with all the radio frequency (RF) power circuitry on an arm of an industrial-like robot. This solution is, however, not practical. Because of the weight of a complete accelerator, the robot would have to weight approximately 3 tons to keep balance.

A viable solution is to integrate the subsystems of an accelerator with the mechanical structures of the robot. In doing so, the whole construction could be balanced in more natural way while the weight could be kept at an acceptable level. Such a solution is nonetheless much more difficult to develop as it demands precise alignment and simultaneous rotations of two independent mechanical setups - the supporting structure of the robot and subsystems of the accelerator, in particular the microwave rotary joints.

After an initial study of the problem, a general idea for the construction emerged and a number of basic requirements and assumptions were made in respect to the mechanics and the RF systems. It was assumed that the demonstrator should be compatible with any surgical table and its height in working position should not exceed $3 \mathrm{~m}$, while in transport position should not exceed $2 \mathrm{~m}$ in order to fit into any doorways. The width of the unit was restricted to $1.25 \mathrm{~m}$. As to the range of rotation of the therapeutic head, the rather wide limits were set: from $-45^{\circ}$ to $+45^{\circ}$ for crosswise rotation, $30^{\circ}$ of maximum inclination toward the crane and $45^{\circ}$ outward the crane, crane rotation in a range from $-90^{\circ}$ to $+90^{\circ}$ (the nomenclature of the movements is explained in Fig. 1). Thanks to the wide ranges of rotations of the segments of the robot, the machine offers large working space for positioning the radiative head, as illustrated in Fig. 2.

At an early stage of the design, the rough layout of the most important and bulky accelerator components such as the accelerating structure, magnetron, RF power divider, circulator, water load, magnetron modulator, and the electron gun modulator, within the robot structure was worked

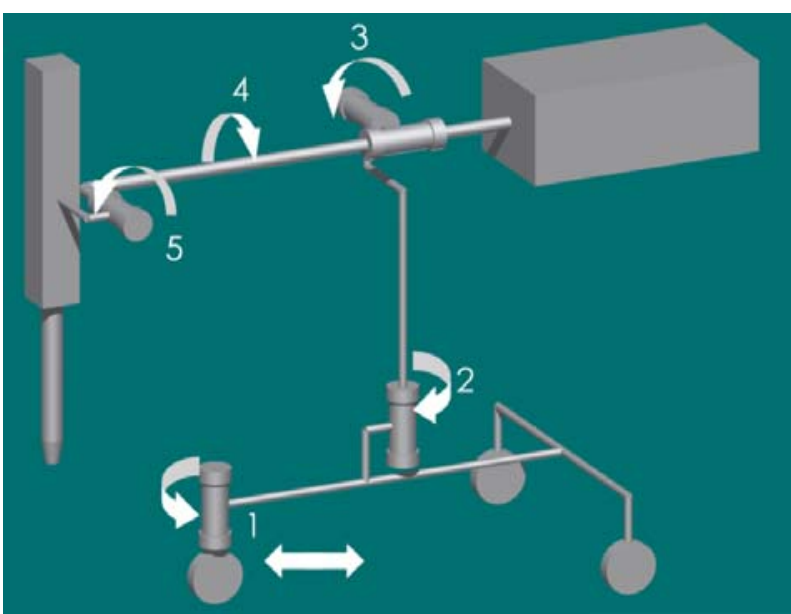

Fig. 1. Kinematical scheme of the IOERT demonstrator. In this solution the accelerator and the robot are integrated in one construction. The nomenclature used for movements is as follows: (1) troley drive and steering, (2) column rotation, (3) crane inclination, (4) crane rotation, (5) head inclination. 

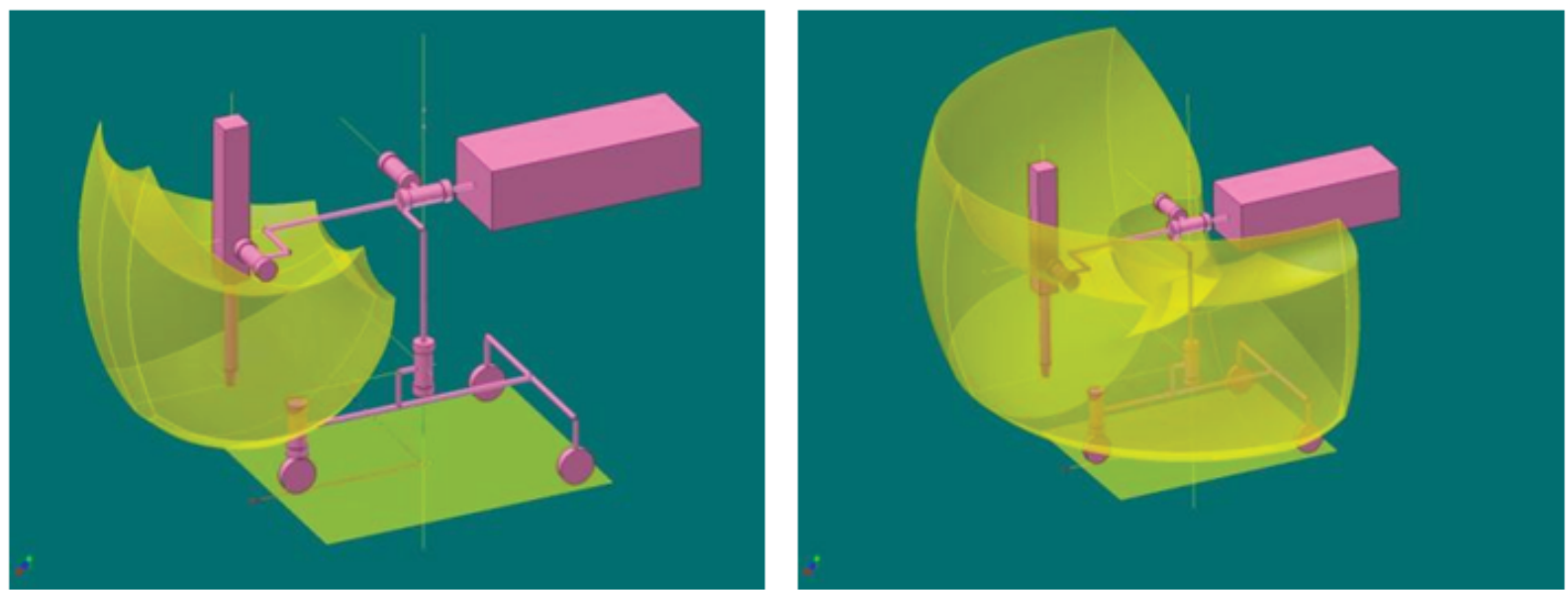

Fig. 2. Working space of selected kinematical scheme. Left panel: realized by rotary joints 3, 4 and 5 only. Right panel: realized by rotary joints $2,3,4$ and 5 .
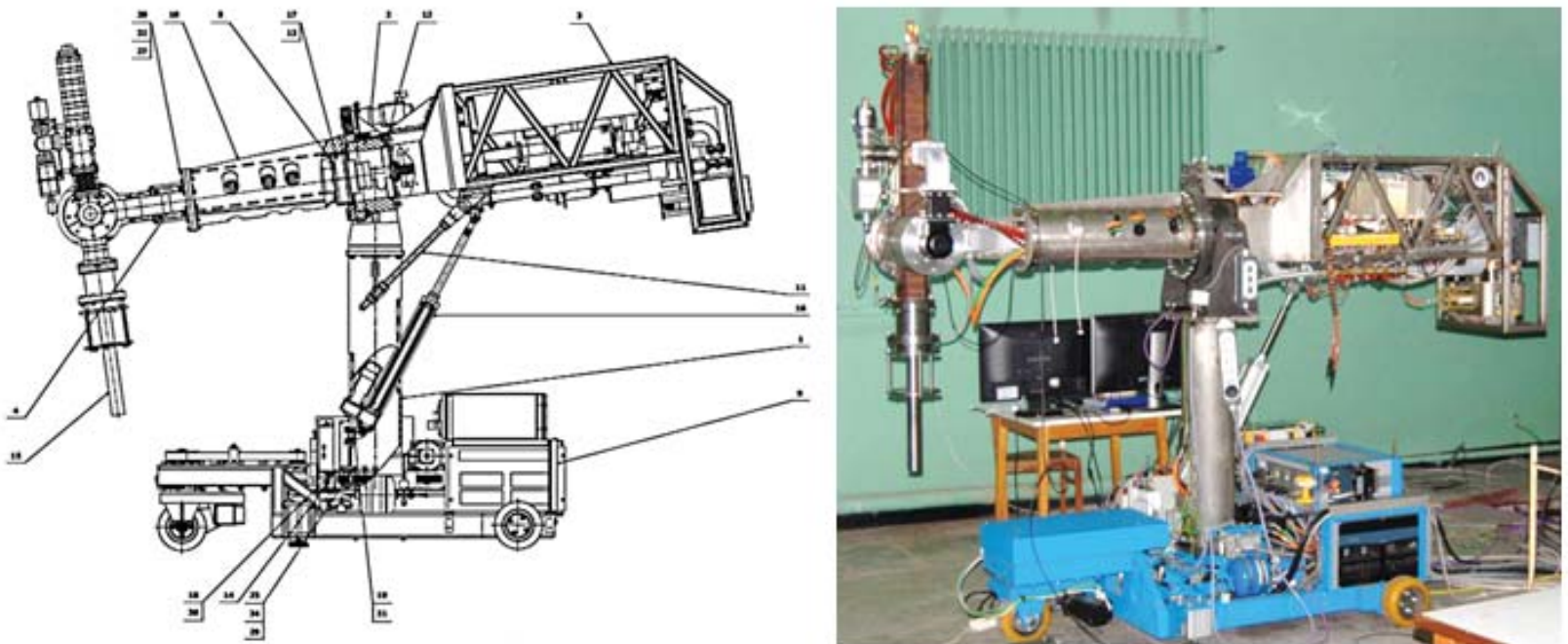

Fig. 3. Example of 2D mechanical documentation of the entire demonstrator (left). Photograph of the assembled machine (right).

out. By means of the computer modeling, the most appropriate kinematical scheme was selected and further optimized. Figure 1 shows a model used for studies of the kinematics. Based on a similar model, the forces and torques in the structure were studied. This resulted in determination of required strength of the construction as well as allowed to balance the weight on both ends of the structure by adjustment of locations of various components within the construction. Once the knowledge of forces and torques was gathered, the supporting structure was designed and proper bearings and drives were selected. In the next step, the auxiliary systems such as electrical, control, and cooling were designed.

After completing the technical documentation, various components were prototyped and tested. Finally, the demonstrator was manufactured and assembled. The tests of the demonstrator are underway, as described further. Figure 3 shows an example of 2D mechanical documentation of the entire demonstrator as well as a photograph of the assembled machine.

The novel solutions developed for the robot are the subject of two patents awarded to the NCBJ and one pending patent application filed at the Patent Office.

\section{Development of the accelerating structure and RF power system}

The accelerating structure designed for the demonstrator is a standing-wave, axially coupled $\pi / 2$ mode structure operating at a resonance frequency of $2.9985 \mathrm{GHz}$. The construction of the structure started with 2D and 3D RF modeling aimed at optimization of the cavity shape. For the RF calculations, the Superfish and the CST Microwave Studio codes were used. The calculated electromagnetic (EM) fields were then used as an input for the electron beam dynamics calculations made with the General Particle Tracer code. Several variants of the structure with different number of resonator cavities were studied in order to find an optimal compromise between the structure length and the RF power consumption. The final design calls for an 18-cavity structure, as illustrated in a 3D technical drawing in Fig. 4.

The resonators were manufactured on site using precise computer numerical control (CNC) machining. After RF measurements of each resonator, the structure was assembled and welded under vacuum. The RF tuning was performed before the final thermal processing. Figure 5 presents a pho- 


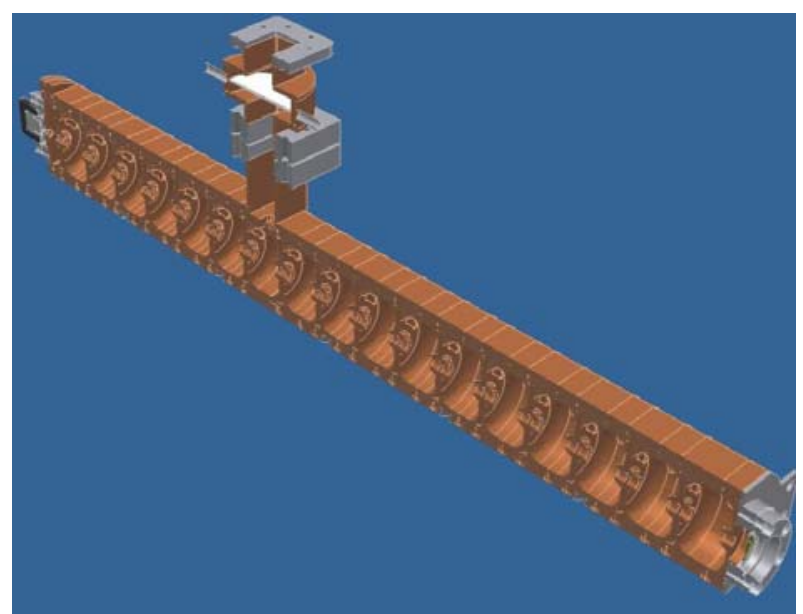

Fig. 4. Example of 3D mechanical documentation of the accelerator structure.

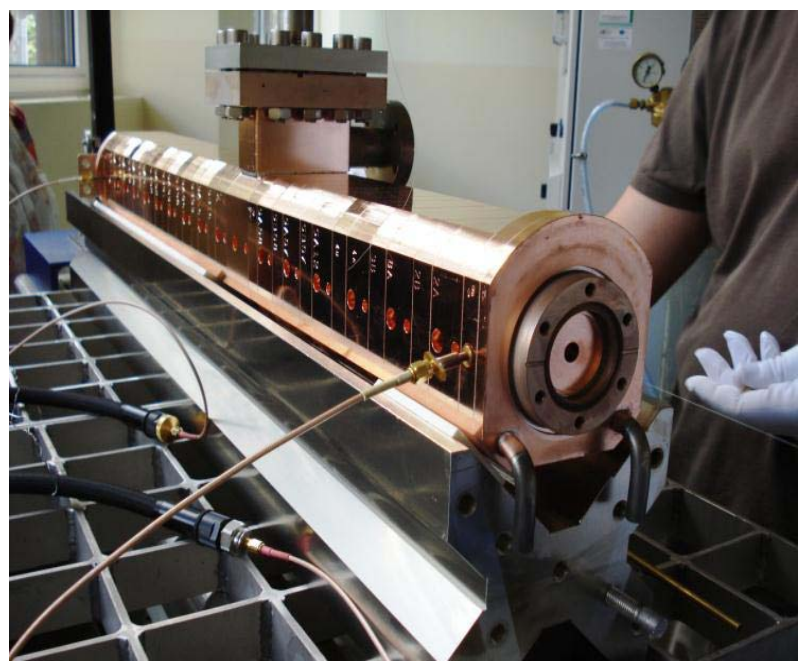

Fig. 5. Photograph of the accelerator structure during RF measurements.

tograph of the assembled structure during the RF measurements.

The accelerating structure is equipped with a triode electron gun. The length of the structure is $940 \mathrm{~mm}$ and the weight is $53 \mathrm{~kg}$.
Parallel to the design of the accelerating structure, the RF power system was designed. Figure 6 illustrates a model of the system. The RF power source is a magnetron. The RF power is then distributed to the accelerating structure via hard waveguides. The RF system includes two rotary joints, a RF power divider, serving as a means of adjusting the power level, and a number of RF diagnostic and control devices.

The structure is equipped with a triode electron gun. The first model of the accelerating structure delivers electron beams in energy range from $2.5 \mathrm{MeV}$ to about $9 \mathrm{MeV}$.

\section{Development of the beam-forming system}

The beam-forming system can be viewed as an interface between the electron accelerator and the patient. The system has several functions. First, it serves to transform the primary electron beam into a therapeutically suitable beam. The primary beam that leaves the accelerating structure is focused on the exit window to a small spot of a diameter about $3 \mathrm{~mm}$ and has a Gaussian-like intensity distribution. To be of clinical use, the beam, however, has to be uniformly distributed over much larger area at the patient plane. In an optimal radiotherapy, the radiation dose should be delivered exclusively to the target area of the patient body with no dose outside this area. Although the perfect case is not practically realizable, it is an important role of the beam-forming system to collimate the therapeutic beam to the target area so that the healthy tissues located outside the field are spared as much as possible. Moreover, in the case of a mobile accelerator specialized for the IOERT, the construction of the beam-forming system has to be lightweight and compact, which implies simplicity. Yet the delivered beam should conform to the strict requirements of quality and safety standards $[4,5]$. To find an optimal solution for the construction of the beam-forming system, Monte Carlo modeling was extensively employed. Various aspects of the development works have been reported elsewhere [6-8].

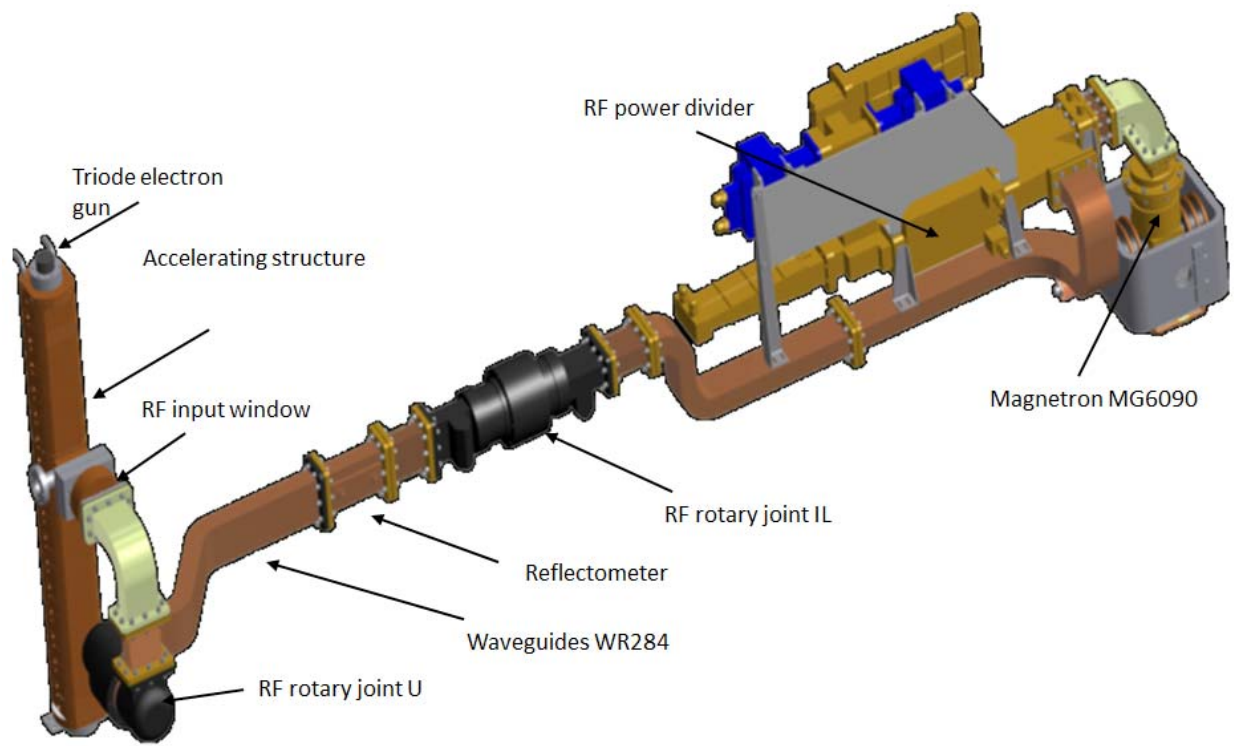

Fig. 6. Model of the RF power system. 
The final construction of the demonstrator beam forming system employs scattering and flattening foils, primary and secondary collimator and an ionization chamber. Those elements comprise one assembly that has no moving parts and is attached to the accelerator right next to the beam exit window. The foils are universal for all beam energies and field sizes. In an IOERT procedure, the field size is finally determined only right before the irradiation once the tumor is surgically removed. Technically, the beam is limited to the target area by means of an applicator. In the IOERT, the electron beam applicators are thin-walled tubes that are at one end inserted into the tumor lodge and at the other end attached to the accelerator right next to the last fixed collimator. The diameter of the applicator is selected for each patient individually. The material and thickness of the applicator is designed such that the leakage electron radiation is below the respective limits suggested by the safety standard [5]. Typically, manufacturers of the IOERT accelerators provide applicators with diameters ranging from 3 to $10 \mathrm{~cm}$ with $1 \mathrm{~cm}$ step. For successful therapy, it is necessary to align the axis of the applicator with the axis of the accelerator. The aligning procedure is called docking. In practice, once the applicator is inserted into the surgical site in the body, the accelerator radiative head is positioned so that its axis overlaps with the applicator axis. Generally, there are two schemes of docking (i) the hard docking in which the tip of the collimator assembly is directly connected with the upper end of the applicator and (ii) the soft docking in which an air gap of certain width is left between the applicator's upper end and the collimator assembly. Each solution has its advantages and weaknesses and it is not possible to fairly assess which of the two solutions is better. Soft docking is superior in terms of patient safety from injury in case of failure in accelerator positioning; however, it is much more difficult to align the axis of the applicator and accelerator. On the contrary, in hard docking, the axis alignment is achieved easily and naturally since both elements are in tight contact. On the other hand, this raises some concerns in respect of patient safety. For hard docking to be safe, the accelerator movements have to be very gentle and precise, which is somewhat challenging to achieve in a machine weighting a few hundreds

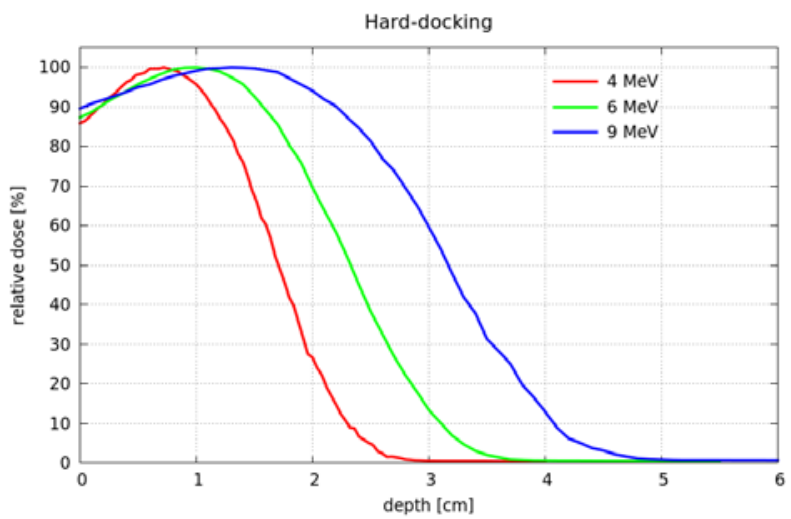

kilograms. Our demonstrator was designed such that both docking schemes are possible without any need for modifications in the beam-forming system. Thanks to such a solution, the future end users of the accelerator will be offered freedom in selection of the applicator docking scheme according to their individual preference.

To study the performance of the beam-forming system in both the docking schemes, experimental versions of the applicators were produced in four diameters ranging from 4 to $12 \mathrm{~cm}$. The applicators are made of steel and have variable wall thickness in the range of 1.2-1.7 $\mathrm{mm}$, depending on the diameter (thicker walls for larger diameter). All applicators are of circular cross-section. It was, however, shown, by means of the Monte Carlo calculation, that the machine is capable of delivering well-formed therapeutic beam also for applicators of noncircular shape. Applicators of oval or rectangular cross-sections would potentially be very useful in the IOERT therapy of certain organs.

\section{Results}

Up to now, the demonstrator has been manufactured and assembled. The performance tests are underway with very promising preliminary results. The robot performs as expected. The movements of all segments are smooth, gentle, and of controllable speed, which is a prerequisite for safe docking of applicators. In the demonstrating version of the robot, movement of each drive is controlled separately form a control pendant. Up to now, the performance of the accelerator was tested in the beam energy range from 4 to $9 \mathrm{MeV}$. The dose distribution in water was measured at three different settings of beam energy and at four diameters of the circular applicators (3, 5,10 , and $12 \mathrm{~cm}$ inner diameter). The preliminary results obtained with the PTW MP3 water phantom and Mephysto software are presented in Figs. 7 to 9. Percentage depth dose and off-axis profiles were measured with the PTW diode E field detector and the PTW semiflex ionization chamber as a reference detector. Noteworthy are relatively high surface doses close to about $90 \%$ of the dose maximum and well flattened off-axis profiles. Contrary to external beam radiotherapy where the dose on the

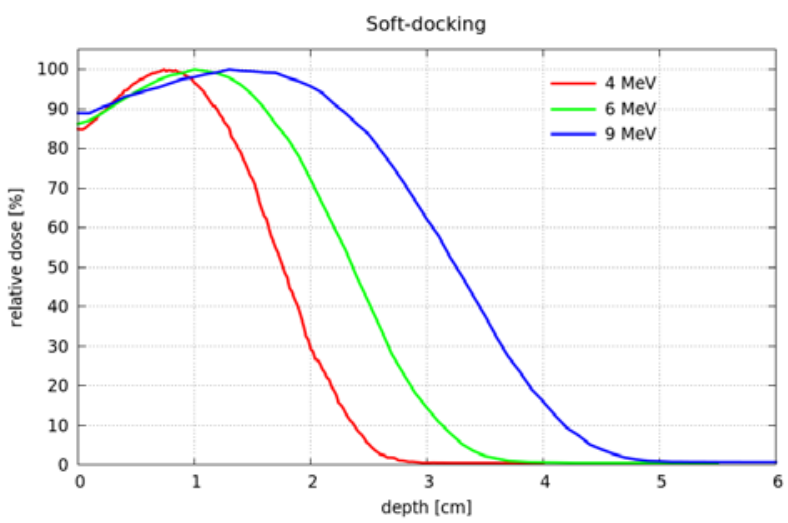

Fig. 7. Measured percentage depth doses in water at 4, 6 and $9 \mathrm{MeV}$ nominal electron beam energy. Left panel for a hard docking applicator of $10 \mathrm{~cm}$ diameter; right panel for a soft docking applicator with $10 \mathrm{~cm}$ diameter. 

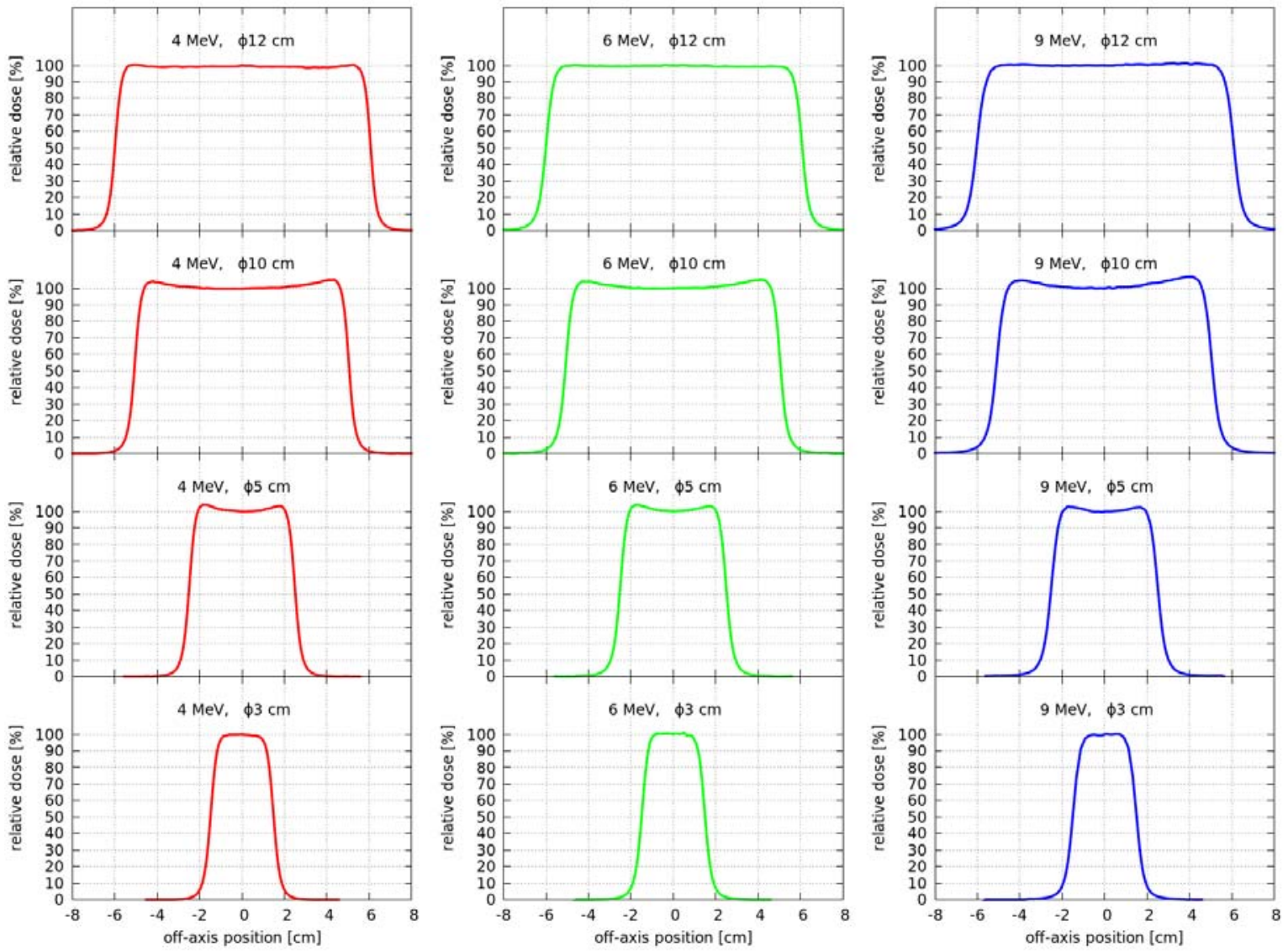

Fig. 8. Off-axis profiles measured with hard-docked applicators at 4, 6 and $9 \mathrm{MeV}$ electron beam energy (left, center and right column, respectively). The measurements were done with applicators of diameters $12 \mathrm{~cm}$ (top row), $10 \mathrm{~cm}$ (second row), $5 \mathrm{~cm}$ (third row) and $3 \mathrm{~cm}$ (bottom row).
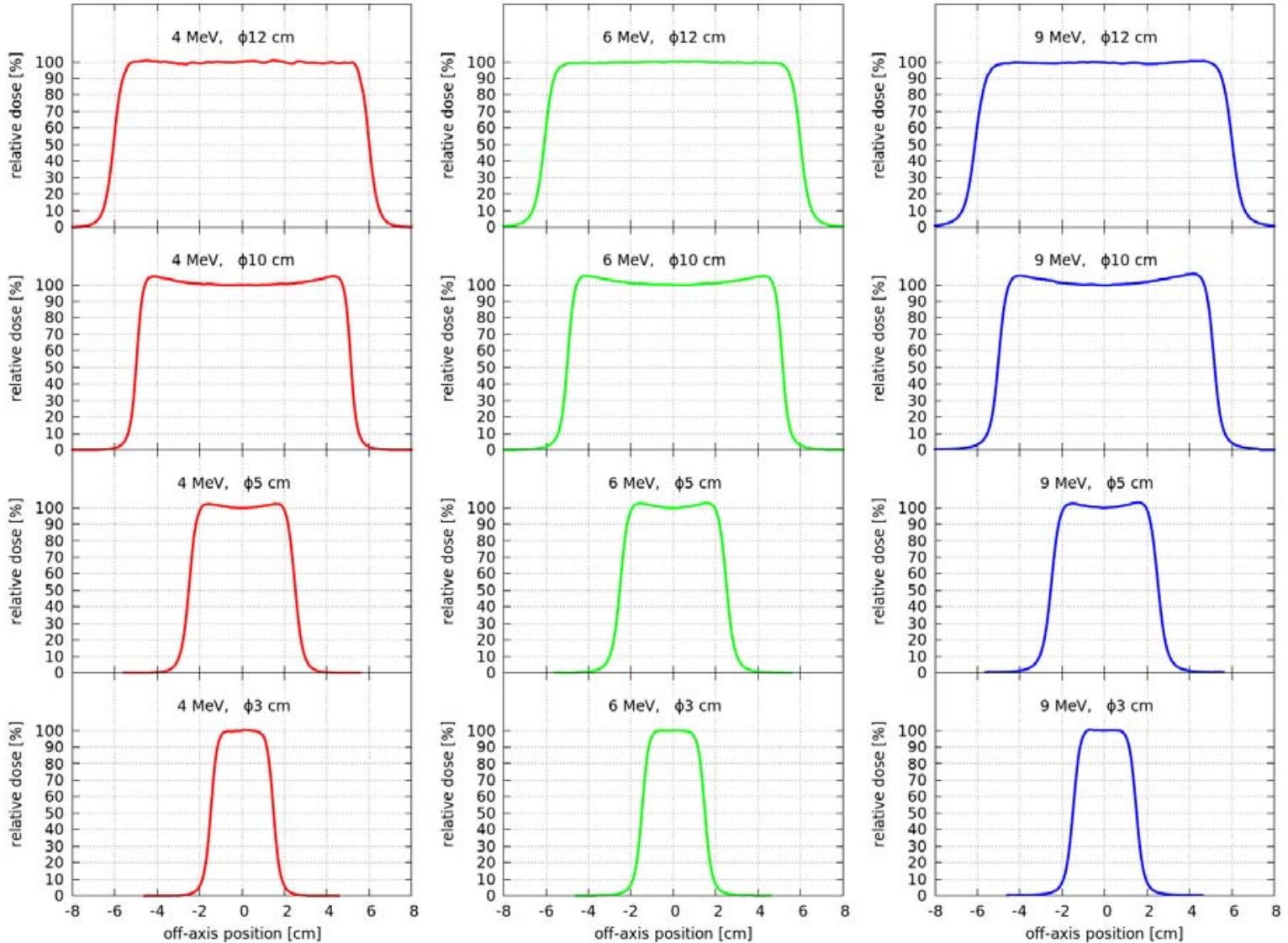

Fig. 9. The same as in Fig. 8 but for soft-docked applicators. 
skin should be minimized, in the IOERT, the high surface dose is of advantage due to the fact that there is a direct access to the target as the skin is surgically removed from the field during irradiation. Preliminary measurements of the leakage radiation outside the field were performed as well indicating that the level of leakage is significantly below the requirements of the IEC standard.

\section{Outlook}

The results obtained so far prove that all major components of the demonstrator are performing correctly. These results also form the basis of our next project aimed at further development of the technology and in particular on producing a preclinical prototype. This project is funded by the National Centre for Research and Development (NCBR) and is already ongoing under an acronym Intra-Dose. Contrary to the demonstrator development, which was realized solely by the NCBJ, the new project is being carried out in a consortium with the Greater Poland Cancer Centre (WCO) and two industrial partners. Within the framework of the Intra-Dose project, the technology developed within the AiD project is going to be used as a base for a machine with extended functionality in terms of the user interface. In particular, the aims are to develop a user-friendly steering of the robot, to make the machine lighter in terms of weight and to construct applicators that could be applied in a clinical environment. In addition, the energy range of the accelerator is going to be extended up to $12 \mathrm{MeV}$.

Acknowledgments. The work was done in Otwock-Świerk, Mazovia, Poland. The work on the first model of the accelerator was supported by the EU Structural Funds, project no. POIG.01.01-14-012/08-00. Further work was supported by the National Research and Development Centre, Poland in the scope of 2nd edition of the Applied Research Programme, contract no. PBS2/B9/26/2014.

\section{References}

1. Polgár, C., Van Limbergen, E., Pötter, R., Kovács, G., Polo, A., Lyczek, J., Hildebrandt, G., Niehoff, P., Guinot, J. L., Guedea, F., Johansson, B., Ott, O. J., Major, T., \& Strnad, V. (2010). Patient selection for accelerated partial-breast irradiation (APBI) after breast-conserving surgery: recommendations of the Groupe Européen de Curiethérapie-European Society for Therapeutic Radiology and Oncology (GEC-ESTRO) breast cancer working group based on clinical evidence. Radiother. Oncol., 94(3), 264-273. DOI: 10.1016/j.radonc.2010.01.014.

2. ASTRO Task Force. (2009). Accelerated partial breast irradiation consensus statement from the American Society for Radiation Oncology (ASTRO). Int. J. Radiat. Oncol. Biol. Phys., 74(4), 987-1001. DOI: 10.1016/j.ijrobp.2009.02.031.

3. National Centre for Nuclear Research. (January 2014). Accelerators \& detectors. Retrieved September 8, 2014, from http://aid.ncbj.gov.pl/index.php/en.

4. Kania, M., \& Rostkowska, J. (2001). Krajowe zalecenia dotyczące kontroli aparatów stosowanych w teleradioterapii. Pol. I. Med. Phys. Eng., 7(3), 199-278.

5. European Committee for Electrotechnical Standardization. (2009). European Standard: Medical electrical equipment. Part 2-1: Particular requirements for the safety of electron accelerators in the range of $1 \mathrm{MeV}$ to $50 \mathrm{MeV}$. IEC 60601-2-1 Ed. 3.0. Brussels.

6. Adrich, P., Baczewski, A., Baran, M., Drabik, W., Gryn, K., Hanke, R., Jakubowska, E., Jankowski, E., Kędzierski, G., Kielar, N., Kujawski, Ł., Kopeć, J., Kosiński, K., Kozioł, R., Kraszewski, P., Krawczyk, P., Kulczycka, E., Lalik, P., Marczenko, M., Masternak, A., Misiarz, A., Olszewski, J., Ozon, K., Pławski, E., Polak, A., Psonka, W., Rutkowska, M., Rzadkiewicz, J., Sienkiewicz, Z., Staszczak, M., Swat, K., Syntfeld-Każuch, A., Terka, M., Wasilewski, A., Wilczek, J., Wojciechowski, M., Wójtowicz, M., Wronka, S., Wysocka-Rabin, A., \& Zalewski, K. (2014). A new mobile electron accelerator for intra operative electron radiation therapy. Int. J. Mod. Phys. Conf. Ser., 27, 1460125. DOI: 10.1142/S2010194514601252.

7. Wysocka-Rabin, A., Adrich, P., \& Wasilewski, A. (2014). Radiation protection studies for a new mobile electron accelerator for intra operative radiation therapy (IORT). Prog. Nucl. Sci. Technol., 4, 298-302.

8. Wysocka-Rabin, A., Adrich, P., \& Wasilewski, A. (2011). Monte Carlo study of a new mobile electron accelerator head for intra operative radiation therapy (IORT). Prog. Nucl. Sci. Technol., 2, 181-186. 